INPLASY

PROTOCOL

To cite: Yang et al. The Safety and Efficacy of Entecavir Associated with Adefovir Dipivoxil Versus Tenofovir Disoproxil Fumarate in the Treatment of Chronic Hepatitis B Patient with Adefovirresistant: a protocol for systematic review and metaanalysis. Inplasy protocol 202110006. doi:

10.37766/inplasy2021.1.0006

Received: 2 January 2021

Published: 2 January 2021

Corresponding author: Wen-ce Zhou

zhouwenceldyy@163.com

Author Affiliation:

Department of general surgery, the first hospital of Lanzhou University, Lanzhou, China

Support: None.

Review Stage at time of this submission: Preliminary searches.

Conflicts of interest: None.

\section{The Safety and Efficacy of Entecavir Associated with Adefovir Dipivoxil Versus Tenofovir Disoproxil Fumarate in the Treatment of Chronic Hepatitis B Patient with Adefovir-resistant: a protocol for systematic review and} meta-analysis

Yang, SJ1; Li, X2; Dong, S3; Shi, ZL4; Zhang, H; Zhou, WC6.

Review question / Objective: Population: CHB patients with detectable serum HBV DNA levels and genotypically confirmed resistance mutations to ADV; Intervention: the combination of ETV and ADV; Comparator: the TDF monotherapy; Outcome: Biochemical function, HBV serum markers, HBV DNA load, and liver ultrasonography were performed at treatment initiation and during the weekends following weeks 12, 24, 36, 48, 60, 72, 84 and 96; Studies: RCT, cohort study and case-control study.

Information sources: A systematic search of online databases including PubMed, EMBASE, Web of science and Cochrane Library will be performed until the end of December 2020 using related search terms, including "chronic hepatitis B", "adefovir dipivoxil", "entecavir" and "tenofovir disoproxil fumarate". In addition, congress and conference proceedings will be manually retrieved. Related articles and references of included research will also be tracked to find potential studies. If significant data was incomplete in included study, we will contact the authors to get unpublished data.

INPLASY registration number: This protocol was registered with the International Platform of Registered Systematic Review and Meta-Analysis Protocols (INPLASY) on 2 January 2021 and was last updated on 2 January 2021 (registration number INPLASY202110006).

\section{INTRODUCTION}

Review question / Objective: Population: CHB patients with detectable serum HBV DNA levels and genotypically confirmed resistance mutations to ADV; Intervention: the combination of ETV and ADV; Comparator: the TDF monotherapy; Outcome: Biochemical function, HBV serum markers, HBV DNA load, and liver 
ultrasonography were performed at treatment initiation and during the weekends following weeks $12,24,36,48$, 60, 72, 84 and 96; Studies: RCT, cohort study and case-control study.

Condition being studied: The World Health Organization estimates that about 400 million patients globally are actively infected with hepatitis B virus (HBV), which is a dangerous virus that can generate severe liver disease including chronic hepatitis B (CHB), liver failure, cirrhosis, and hepatocellular carcinoma. Through the inhibition of virus replication or virus elimination, CHB therapy prevents progress of liver injury, fibrosis, and carcinoma, including nucleotide analogues and interferon alpha. However, drug-resistant HBV, as a mutant selected by long-term antiviral treatments, reduce the efficacy of clinical treatment. The adverse effects and low response rate of the interferon alpha account for $20-40 \%$ of the whole interferon alpha therapy, in addition patients treated with nucleotide increase the risk of drug resistance because the long half-life of covalently closed circular (ccc) DNA. Tenofovir disoproxil fumarate (TDF) and Entecavir (ETV), with high genetic barrier, have potent anti-HBV effects and still keep very low rate of resistance, are recommended for the first-line treatment of CHB in current practice guidelines, especially in patients with drug resistance for virus mutation,Besides, Adefovir dipivoxil (ADV) is also effective drug with a relatively lower drug resistance rate and no cross-resistance.

\section{METHODS}

Participant or population: Inclusion criteria required $\mathrm{CHB}$ patients with detectable serum HBV DNA levels ( $\geq 1.0 \times 103 \mathrm{IU} / \mathrm{mL}$ ) and genotypically confirmed resistance mutations to ADV (rtA181V/T and/or rtN236T mutation). Exclusion criteria include patients with hepatitis delta virus, hepatitis C virus, or HIV coinfection, patients with hepatocellular carcinoma or diabetes and patients with liver cirrhosis.
Intervention: The combination therapy group, treated with ETV and ADV (0.5 and $10 \mathrm{mg}$ per day, respectively).

Comparator: The monotherapy group, treated with TDF (300 $\mathrm{mg}$ per day).

Study designs to be included: RCT, cohort study and case-control study.

Eligibility criteria: Clinical trials to evaluate the safety and efficacy of ETV associated with ADV Versus TDF in the treatment of chronic hepatitis B patient with adefovirresistant, without published year, publication status limitations.

Information sources: A systematic search of online databases including PubMed, EMBASE, Web of science and Cochrane Library will be performed until the end of December 2020 using related search terms, including "chronic hepatitis B", "adefovir dipivoxil", "entecavir" and "tenofovir disoproxil fumarate". In addition, congress and conference proceedings will be manually retrieved. Related articles and references of included research will also be tracked to find potential studies. If significant data was incomplete in included study, we will contact the authors to get unpublished data.

Main outcome(s): Biochemical function, HBV serum markers, HBV DNA load, and liver ultrasonography were performed at treatment initiation and during the weekends following weeks 12, 24, 36, 48, $60,72,84$ and 96 . Besides, all the endpoints reported in the included studies will be collected and evaluated, although we may not mention some of them in this protocol.

Data management: Two reviewers will read the titles and abstracts of all identified records to exclude clearly unrelated records based on the inclusion criteria. Then the full texts of the articles retained were reviewed to further determine their suitability. Any disagreement will be resolved by a third reviewer. We will show the selection process in details in the PRISMA flow chart. Two authors of this review will independently extract the data 
using a pre-defined form. The basic characteristics, related outcome and quality evaluation information of included studies will be collected. Similarly, any discrepancies will be resolved by a third reviewer. Data extracted will include author, year, study type, number of participants, intervention, control, demographics, complications, previous history, biochemical function, HBV serum markers, HBV DNA load, liver ultrasonography and the follow-up time.

Quality assessment / Risk of bias analysis: Included study bias will be independently assessed by two reviewers and any disagreement will be solved by a third reviewer. For randomized controlled trials, we will use the Cochrane risk of bias tools to evaluate potential bias in seven specific domains: (1) sequence generation, (2) allocation concealment, (3) blinding of participants and personnel, (4) blinding of outcome assessment, (5) incomplete outcome data, (6)selective outcome reporting, (7) other bias. For cohort studies and case-control studies, 9-star Newcastle-Ottawa Scale will be applied, which rates studies based on eight criteria in three sources of bias.

Strategy of data synthesis: For dichotomous variables, The Odd Risk (OR) with $95 \%$ confidence intervals $(\mathrm{Cl})$ were calculated from each study. Continuous variables will be presented as Standard Mean Difference (SMD) with $95 \% \mathrm{Cl}$. All endpoints will be combined and performed meta-analysis by using DerSimonian and Laird random effects model. We assessed statistical heterogeneity by using Chi2 test and 12 statistic. We will consider significant heterogeneity when $\mathrm{P}<0.10$ for Chi2 or $12>50 \%$. All primary analyses were performed with STATA v15.1 (Stata Corp, College Station, TX).

Subgroup analysis: We will also conduct subgroup analysis to find more potential information based on pre-set criteria in different follow-up time.
Sensibility analysis: $f$ the heterogeneity is high, we will conduct sensitivity analyses based on follow-up time.

\section{Country(ies) involved: China.}

Keywords: chronic hepatitis B; adefovirresistant; adefovir dipivoxil; entecavir; tenofovir disoproxil fumarate.

Contributions of each author:

Author 1 - Si-jie Yang - The author conceived the idea for this study, designed the meta-analysis and drafted the protocol. Author 2 - Xin Li - The author conceived the idea for this study, provided statistical advice and input, drafted the protocol.

Author 3 - Shi Dong - The author designed the meta-analysis.

Author 4 - Zhi-long Shi - The author provided statistical advice and input.

Author 5 - Hui Zhang - The author reviewed the protocol and provided critical feedback. Author 6 - Wen-ce Zhou - Wen-ce Zhou, the author reviewed the protocol and provided critical feedback. 